\title{
Corneo-Uveal-Retinal Adverse Effects after Single Course of Targeted Anti-Cancer Therapy: Case Report and Review of Literature
}

\author{
Maaly Abdel Halim Abdel Fattah ${ }^{1,2,3 *}$, Tariq Abdulmohsen Al-Anazi ${ }^{2}$, \\ Sara Sohail Kayali ${ }^{3}$, Yasser Saleh Almuzaini ${ }^{4}$ and Faisal Mesfer \\ AlQahtani ${ }^{2}$ \\ ${ }^{1}$ Ophthalmology Department, Cairo University, College of Medicine, Cairo, Egypt \\ ${ }^{2}$ Ophthalmology Department, King Faisal Specialist Hospital and Research Center, \\ Riyadh, Saudi Arabia \\ ${ }^{3}$ Al-Faisal University College of Medicine, Riyadh, Saudi Arabia \\ ${ }^{4}$ Imam Abdulrahman Bin Faisal University, College of Medicine, Riyadh, Saudi \\ Arabia \\ *Corresponding Author: Maaly Abdel Halim Abdel Fattah, Ophthalmology \\ Department, King Faisal Specialist Hospital and Research Center, Riyadh, Saudi \\ Arabia.
}

Received: May 15, 2021

Published: May 22, 2021

(C) All rights are reserved by Maaly Abdel

Halim Abdel Fattah., et al.

\begin{abstract}
Oral anti vascular endothelial growth factors therapeutic agents have been evolving rapidly over the last past decades and became one of the great contributors to advancement of cancer therapy and increasing survival rate of the treated patients.

Sorafenib is FDA approved medications for the treatment of solid tumors.

In this article, we reported the clinical features of the first Saudi patient presenting with corneo-uveal retinal adverse effects with subsequent bilateral irreversible blindness.
\end{abstract}

Keywords: Hepatocellular Carcinoma; Targeted Anti-Cancer Therapy; Sorafenib; Oral Anti-Vascular Endothelial Growth Factor; Ocular Toxicity; Irreversible Blindness

\section{Abbreviations}

OD: Oculus Dexter; OS: oculus Sinister; OU: Oculus Uterque; HCC: Hepatocellular Carcinoma; FMS-like Tyrosine Kinase 3: (FLT3) Inhibitors; KFSH\&RC: King Faisal Specialist Hospital and Research Center; CED: Corneal Epithelial Defect; CSF: Cerebral Spinal Fluid; VEGFR: Vascular Endothelial Growth Factor; PDGFR: PlateletDerived Growth Factor Receptor

\section{Introduction}

Cancer is the second leading cause of death in the United States [1]. Estimates for 2020 predicted over 1.8 million new cases of cancer and over 600,000 cancer-related deaths [1]. Cancer is also a major public health problem with large economic impact, estimated in 2010 to represent approximately $\$ 1.16$ trillion USD annually [2]. This translates into a staggering amount of funding dedicated to research and development of novel cancer therapeutics. There are currently over 3000 US Food and Drug Administration (FDA)- 
approved clinical trials (in phase II and III) investigating a diverse array of cancer treatments [3].

Over the last two decades, cancer treatment has been revolutionized by the development of many targeted anticancer agents and immunotherapy.

Targeted therapy is a type of cancer treatment that uses drugs or other substances to precisely identify and attack certain types of cancer cells without affecting normal cells. Targeted drugs can block or turn off signals that make cancer cells grow or can signal the cancer cells to destroy themselves.

Those targeted anticancer drugs have become an increasingly important tool in the oncologic armamentarium but have also been associated with a diverse spectrum of side effects $[4,5]$.

Sorafenib is an oral multikinase inhibitor drug FDA approved for the treatment of primary kidney cancer, advanced liver cancer, FLT3-ITD positive AML and radioactive iodine resistance advanced thyroid carcinoma. It exerts its action through inhibition of several kinases involved in both tumor cells proliferation and angiogenesis (tumor blood supply) including Raf, VEGFR and PDGFR [6].

Considering the frequent use of these drugs, it is vital that ophthalmologists maintain a high index of suspicion for these adverse effects and provide appropriate treatment, while considering the risks and benefits of discontinuing potentially lifeprolonging therapy in conjunction with patients' oncologists.

We describe the clinical presentation and imaging features of a patient with advanced HCC who developed ophthalmic manifestations complicated by bilateral irreversible blindness after single course treatment with Sorafenib.

\section{Case Report}

A 36- year-old male referred to the medical oncology department at KFSHRC in Riyadh, who was diagnosed with advanced hepatocellular carcinoma based on the clinical and radiological assessment (Figure 1). Upon he received an oral course of sorafenib $400 \mathrm{mg}$ BID for 8 days.

He presented with 2 months history of abdominal discomfort and distension, constipation, lethargy, not associated with weight loss or dizziness. On examination he appeared toxic, not jaundiced, had abdominal distension with moderate ascites with no clinical obvious organomegaly. All other systems were normal.

All laboratory work was reviewed:

- Complete blood count showed high WBC ranged from 11.6917.14, low RBC ranged 3.65-4.42, low hemoglobin ranged from 104-123, high absolute neutrophil ranged from 9.6814.4, low absolute lymphocyte ranged from 0.603to1.21.

- Coagulation profile showed: high PT ranged from 14.9- 16.8 with INR ranged from 1.1 to 1.2 .

- CSF analysis showed clear, colorless appearance with no detected cells.

- Kidney function test revealed low creatinine ranged from 34 - 49 with normal urea.

- Normal levels of sodium, chloride, potassium, and calcium levels.

- Liver function test showed high ALT: 355 - 164, high AST: 231 - 59.5, high alkaline phosphatase 248 - 367.

- Tumor markers showed high Alpha fetoprotein AFP: 13.047.

- Acid Fast bacilli, blood, CSF, gram and urine culture are all negative

- Brucella antigens, Hepatitis Bs antigen, Hepatitis C, Herpes Simplex virus, HIV 1-2 antibody screen, Syphilis TP antibody, Toxoplasmosis IgM and Epstein Bar virus were non-reactive.

- $\quad$ CMV titer was raised from $50 \mathrm{IU} / \mathrm{ML}$ to $321 \mathrm{IU} / \mathrm{ml}$ within 2 weeks of admission.

- Rapid multiplex PCR respiratory influenza A and B virus, Parainfluenza B, Corona virus, Bocavirus, Adenovirus, Rhinovirus, Mycoplasma pneumonia, Legionella pneumophila and Bordetella pertussis were negative.

- COVID-19 PCR is negative.

- C-ANCA, P-ANCA, Anti ds-DNA, Anti SSA, Anti SSB, Anti smith antibodies, Anti ANA, Anticardiolipin, Rheumatic factor are either negative or within normal limits except for complement 3 which was detected high 1.93 .

A triphasic abdominal CT showed: advanced HCC (Figure 1).

Additionally, a plain chest x-ray showed small-sized bilateral pleural effusion with adjacent atelectatic changes/consolidation. No pneumothorax or cardiomegaly was noted (Figure 2). 

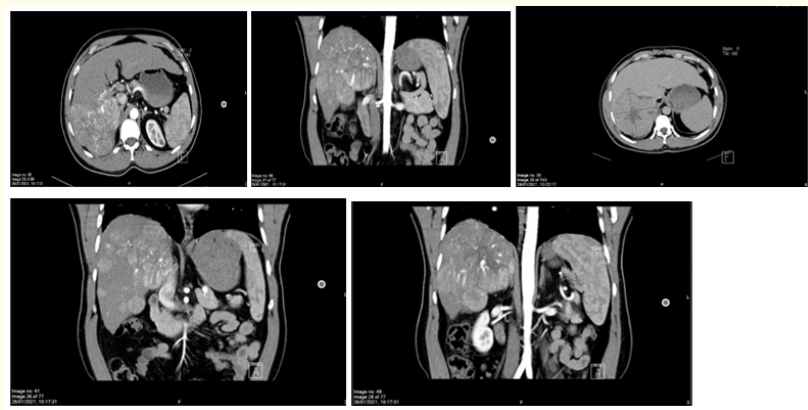

Figure 1: A triphasic abdominal CT showed: advanced HCC.

CT liver arterial phase images, non-contrast, venous, and delayed phase images of the abdomen and pelvis with multiple reformats were taken:

CT report revealed a cirrhotic liver with a nodular surface and fissure widening. Multiple heterogeneous arterial phase hyper-enhancing observations with delayed washout largest are in segment 7 measuring $12.7 \times 10.7 \mathrm{~cm}$, definitely HCC

(LR-5). Most of the lesions are in the right hepatic lobe, however, there is a $2.5 \mathrm{~cm}$ lesion involving the lateral aspect of segment \#4b. The right adrenal gland is inseparable from an adjacent HCC, and direct invasion cannot be excluded nodules.

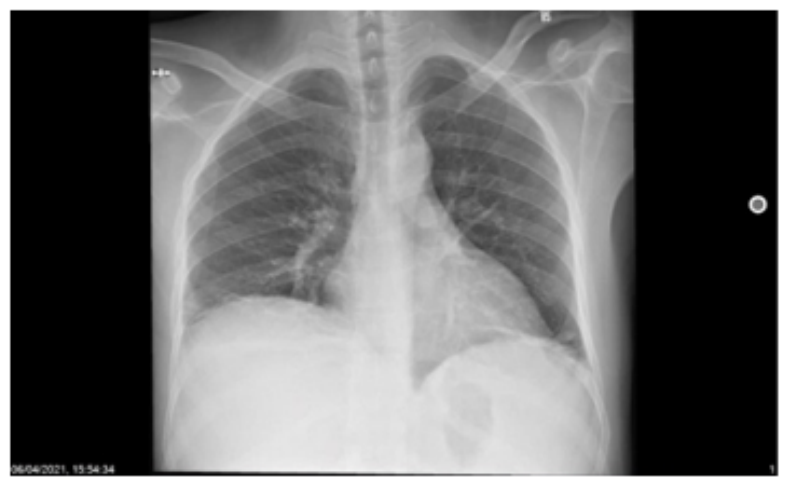

Figure 2: Showed small-sized bilateral pleural effusion with adjacent atelectatic changes/consolidation. No pneumothorax or cardiomegaly.
Brain and orbital MRI revealed no metastatic lesions.

He underwent complete ophthalmological evaluation for profound bilateral visual loss associated with sharp ocular pain 2 days after receiving his 8 days course of Sorafenib, associated with bilateral eye redness, tearing, and photophobia.

Visual acuity of the right eye was Hand motion while counting fingers at 6 feet of the left eye.

Eyes were orthoptic with full extraocular motility bilaterally.

Intraocular pressure (IOP) was $14 \mathrm{mmhg}$ and $11 \mathrm{mmh}$ for the right and left eyes, respectively. But on third day of the follow up, IOP dropped to zero OD and seven OS.

The slit-lamp examination revealed:

- Pupils were non-reactive bilaterally with dense fibrinous membranes extending from the pupil till the anterior surface of the iris leading to a very poor view of the lens and the vitreous and the retina (Figure 3).

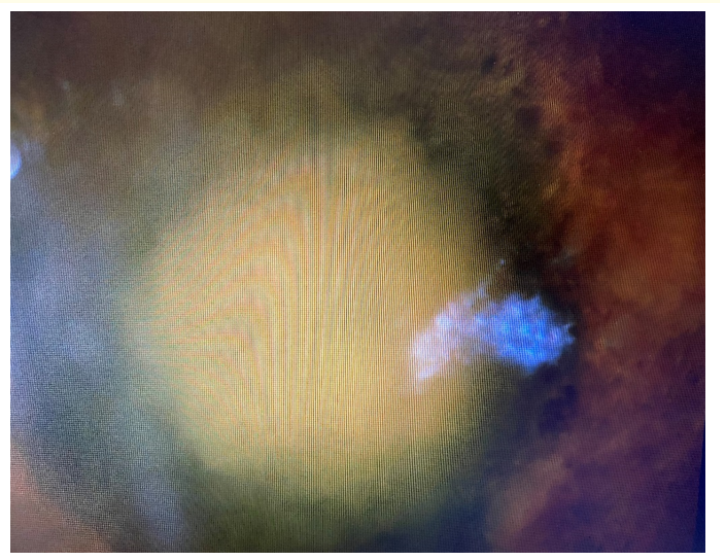

Figure 3: Anterior segment photos of the right eye on presentation.

- The upper and lower eyelids were within normal limits and the conjunctiva was injected at 360 degrees OU. The cornea was edematous with a corneal epithelial defect at the superonasal quadrant measured $3 \mathrm{~mm} \mathrm{~V} * 2 \mathrm{~mm}$ H ellipsoid in shape OD. 
- With a centrally edematous cornea with a large CED involving almost all the cornea surface with no infiltrates OS. No keratitic precipitates OU (Figure 4).

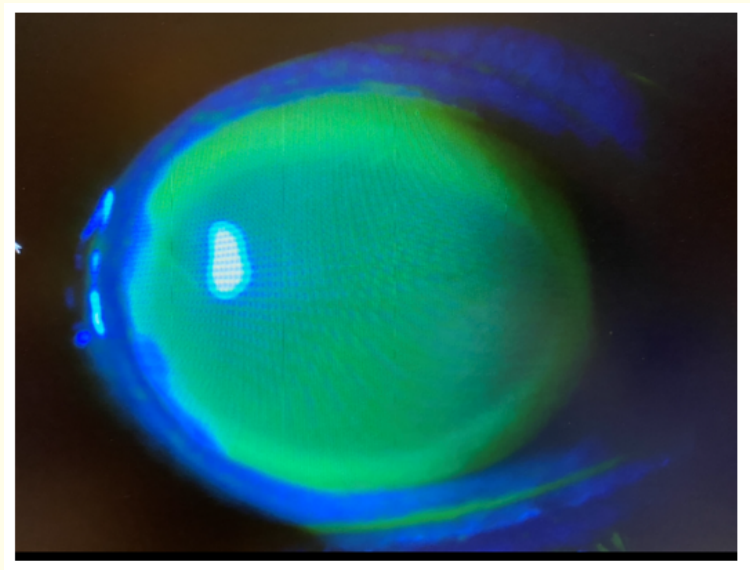

Figure 4: All corneal surface of the left eye stained by fluorescein dye due to the presence of CED.

- No corneal sensation was detected in both eyes.

- The corneal condition of the right eye progressed over the third day to three corneal vertical striae associated with ocular hypotony (Figure 5).

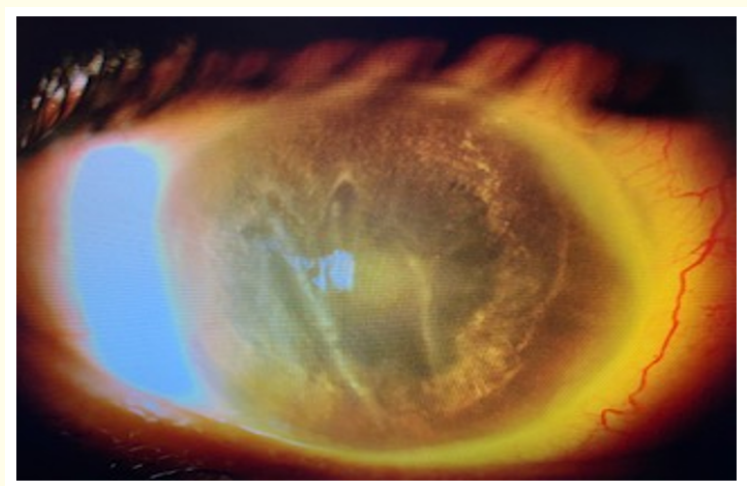

Figure 5: Anterior segment photos of the right eye after 4 weeks: note the striate keratopathy.

- Viral infection (herpes simplex) was suspected due to corneal involvement with loss of the corneal sensation upon topical ganciclovir (Virgan) topical ointment was added.
- But herpes viral infection was excluded by negative laboratory results.

- The anterior chamber showed a +2 flare and +2 cells with an upper, lower, and nasal fibrinous band attached to the pupillary area with a clear lens (Figure 6).

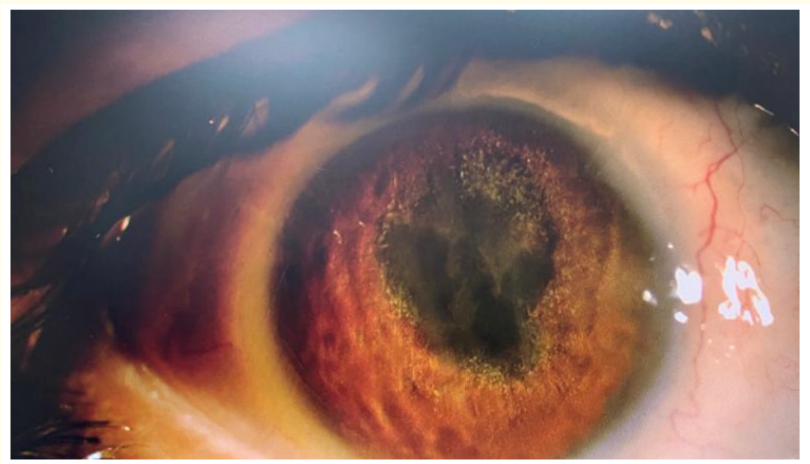

Figure 6: Anterior segment photos of the left eye 4 weeks after presentation.

- Frequent topical corticosteroids associated with cycloplegia (cyclopentolate $1 \%$ and Atropine $0.5 \%$ ) were started.

- The patient was advised to cover his left eye to promote healing of the corneal epithelium.

- Ocular ultrasound showed extensive vitreous opacities with severe choroidal thickening OD more than OS (Figure 7).



Figure 7: Ocular ultrasound of the right eye showing dense vitreous opacities associated with choroidal thickening (third day of the presentation). 
- $\quad$ Systemic corticosteroids (initial Dexamethasone $8 \mathrm{mg}$ IV for 4 weeks then pulse IV methylprednisolone $1 \mathrm{gm}$ daily for 3 days) with Peribulbar triamcinolone acetonide $40 \mathrm{mg} / 1 \mathrm{ml}$ (kenalog) injection OU, were added hopping to improve his ocular functions.

- Unfortunately, over 5 weeks follow up and despite the discontinuation of sorafenib and the use of different lines of steroids (systemic including pulse therapy, peribulbar and topical) the patient did not regain his visual function and discharged in a stable condition.

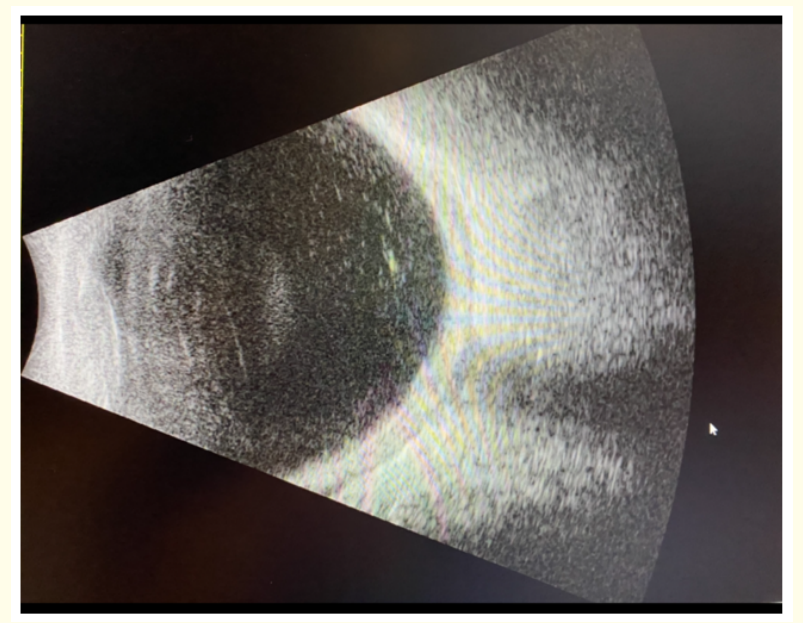

Figure 8: Ocular ultrasound of the left eye showing moderate vitreous opacities associated with moderate choroidal thickening (third day of the presentation).

\section{Discussion}

Three oral anti-vascular endothelial growth factors (anti-VEGF) drugs are widely used: pazopanib (Votrient), sorafenib (Nexavar), and sunitinib (Sutent).

Although each of these medications had slightly variant properties, their main use is in advanced cancer therapy as they block signals inducing cancer cell growth and angiogenesis [7].

Anti- VEGF medications have small molecules which allow them to reach to human retina and choroid. Despite not having research data to support this, it was postulated that the concentration of the daily oral anti-VEGF drug is higher in the uvea than that of a single vitreous injection of the same drug. The medication is found in a much higher concentration in the uvea over a continuous period and remains in the pigmented uvea for several weeks or months $[8,9]$.

Pazopanib, sorafenib, and sunitinib are all mainly used in the treatment of advanced renal cell carcinoma [8].

The most reported non-retinal adverse effects of these medications were eyelid edema, blurred vision, blepharitis, and signs of superficial anterior ocular toxicity which included photophobia, keratitis, dry eye, discharge, ocular irritation, chemosis, and lacrimation, and conjunctival injection [10,11].

Less common non-retinal adverse caused by anti-VEGF medications include discoloration of the eyelashes, extraocular muscle disorders including ptosis, and uveitis although no cases of uveitis were reported with pazopanib use [10].

Retinal adverse effects of these medications include macular edema, papilledema, ischemic optic neuropathy, and vascular events including arterial, venous, or retinal. However, macular edema is not a reported side effect of pazopanib while optic nerve pathologies like ischemic optic neuropathy and papilledema, retinal detachment, and tears are not reported as adverse effects of sorafenib [10,12-14].

A review article by Fraunfelder and Fraunfelder summarized reported ocular adverse effects that developed with sorafenib use, which included: 100 cases of blurred or decreased vision, 46 cases of superficial anterior segment ocular irritation, 33 cases of periocular or ocular edema, 32 cases of conjunctival, vitreal, or retinal bleeding, 19 cases of vascular events including arterial, venous, or retinal occlusions, 17 cases of extraocular muscle disorders like ptosis, 9 cases of retinal detachment or tear, 8 cases of uveitis, 3 cases of macular edema, and one case of eyelash discoloration $[10,12-14]$.

Additionally, Bhatia., et al. reported one case of cataract and blurred vision after sorafenib administration in combination with carboplatin and paclitaxel for metastatic uveal melanoma [15].

Li., et al. reported one case of unilateral central retinal vein occlusion 17 months after sorafenib administration for metastatic 
renal cell carcinoma. Sorafenib discontinuation was required and treatment with mecobalamin was done [16].

Likewise, Szczepanik and Kecik reported one case of bilateral central retinal vein occlusion over 6 months of sorafenib administration for disseminated metastatic renal cell carcinoma. Treatment with enoxaparin and laser photocoagulation was achieved [17].

Gaertner., et al. reported one case of three retinal tears after sorafenib use for the treatment of unresectable hepatocellular carcinoma. The patient complained of blurry vision of the right eye associated with flashes without irritation or pain [14].

Hussain., et al. reported one case of eyebrow loss that occurred 6 weeks after treating iodine refractory thyroid carcinoma with sorafenib 400 my twice daily. Sorafenib was discontinued [18].

As per our knowledge that there were no published case reports to have a combined bilateral pan uveitis with disturbed corneal sensation and chorioretinal adverse effects with the use of Sorafenib.

\section{Conclusion}

The proliferation of targeted anticancer agents has revolutionized cancer treatment and resulted in improved disease-free survival in several difficult-to-treat malignancies. Targeted drugs have become an increasingly important treatment tool, as demonstrated by their growing list of indications. Unfortunately, targeted agents had adverse events of their own, including ocular side effects. Ophthalmologists should maintain a high index of suspicion for those ocular side effects in patients with a history of cancer who have been treated with targeted therapy.

\section{Acknowledgments}

Anoud Faisal Alsaati, Department of Ophthalmology at prince Sultan Military Medical City

\section{Ethical Approval and Consent to Participate}

Our patient signed consent to participate in the study.

\section{Conflict of Interest}

We have no conflicts of interest to disclose.

\section{Consent}

Written informed consent was obtained from the family of the patient for publication of this case report and the accompanying images. A copy of the written consent is available for review by the editor of this journal.

\section{Bibliography}

1. Siegel RL., et al. "Cancer statistics, 2020". CA: A Cancer Journal for Clinicians 70.1 (2020): 7-30.

2. Rehman FU., et al. "Anticancer therapeutics: a brief account on wide refinements". American Journal of Cancer Research 10.11 (2020): 3599-3621.

3. Dembic Z. "Antitumor drugs and their targets". Molecules (2020).

4. Dalvin LA., et al. "Checkpoint Inhibitor Immune Therapy: systemic indications and ophthalmic side effects". Retina 38.6 (2018): 1063-1078.

5. Fortes BH., et al. "Ophthalmic adverse effects of immune checkpoint inhibitors: The Mayo Clinic experience". British Journal of Ophthalmology (2020).

6. Roberts PJ and Der CJ. "Targeting the Raf -MEK-ERK mitogenactivated protein kinase cascade for the treatment of cancer". Oncogene 26 (2007): 3291-310.

7. King J., et al. "Sorafenib for the Treatment of Advanced Hepatocellular Cancer - a UK Audit". Journal of Clinical Oncology 29.4 (2017): 256-262.

8. Imperia PS., et al. "Ocular complications of systemic cancer chemotherapy”. Survey of Ophthalmology 34 (1989): 209-230.

9. Fortes BH., et al. "Ocular Toxicity of Targeted Anticancer Agents". Drugs (2021).

10. FT Fraunfelder. "FW Fraunfelder". Journal of Ocular Pharmacology and Therapeutics (2018).

11. Harman LE. "Ophthalmic complications related to chemotherapy in medically complex patients". Cancer Control 23 (2016): 150-156.

12. Wegner A and Khoramnia R. "Neurosensory retinal detachment due to sunitinib treatment". Eye 25 (2011): 1517-1518.

13. Hasan R. "A retinal tear induced by pazopanib therapy: a case report". Medical Education Forum 16 (2015): 1-3. 
14. Gaertner KM., et al. "A case of retinal tear associated with use of sorafenib". Frontiers in Oncology 4 (2014): 1-2.

15. Bhatia S., et al. "Phase II trial of sorafenib in combination with carboplastin and paclitaxel in patients with metastatic uveal melanoma: SWOG S0512". PLoS ONE 7.11 (2012): e48787.

16. Li ZY., et al. "Metastatic renal cell carcinoma: the first report of unilateral fundus hemorrhage induced by sorafenib". Oncotarget 7.23 (2016): 35181-35187.

17. Szczepanik S and Kecik D. "Bilateral central retinal vein occlusion in a patient with disseminated metastatic renal cell carcinoma treated with sorafenib". Retinal Cases and Brief Reports 6.2 (2012): 148-150.

18. Hussain SZ., et al. "Development of skin hypopigmentation in a patient with metastatic papillary carcinoma thyroid treated with Sorafenib". BMC Endocrine Disorders 13 (2013): 29.

\section{Volume 4 Issue 6 June 2021}

(C) All rights are reserved by Maaly Abdel Halim Abdel

Fattah., et al. 